\title{
A doença crônica orgânica na infância e as práticas educativas maternas
}

\author{
Cesar Augusto Piccinini \\ Elisa Kern Castro \\ Patrícia Alvarenga \\ Silvia Vargas \\ Viviane Z. Oliveira \\ Universidade Federal do Rio Grande do Sul ${ }^{1}$
}

\begin{abstract}
Resumo
Estudos apontam que a presença de doença crônica orgânica na infância pode se constituir num importante fator de mediação da qualidade da interação mãe-criança. Entretanto, poucos estudos investigam as práticas educativas maternas em crianças portadoras de doença crônica. Os cuidados diários exigidos pelo tratamento da enfermidade, bem como a fragilidade física e emocional da criança podem afetar as práticas educativas maternas. Participaram do estudo 40 mães, metade das quais eram mães de crianças portadoras de doença crônica orgânica e as demais de crianças sem doença crônica. As crianças eram de ambos os sexos e tinham entre 03 e 05 anos de idade. Os participantes foram recrutados em hospitais e pré-escolas públicas. Uma entrevista semi-estruturada foi utilizada para se investigar as práticas educativas maternas, examinadas através de análise de conteúdo. Os resultados revelaram uma tendência marginalmente significativa no grupo com doença crônica a utilizar um número menor de práticas coercitivas. Além disso, houve uma diferença significativa entre os grupos quanto ao uso de punição física e privação/castigo, indicando que as mães de crianças com doença crônica utilizaram com menor freqüência este tipo de prática. Os resultados apóiam a expectativa inicial de que as mães de crianças com doença crônica seriam menos coercitivas quando comparadas às mães do outro grupo.
\end{abstract}

Palavras-chave: práticas educativas maternas; doença crônica infantil; infância

\begin{abstract}
The physical chronic disease in childhood and the mother's childrearing practices. . Studies indicate that the presence of physical chronic disease in childhood may constitute an important factor to the quality of mother-child interaction. However, few studies investigate the childrearing practices in children with physical chronic disease. The daily care demand by the disease treatment as well as the child physical and emotional fragility may affect the childrearing practices. Fourty mothers participated in this study, half of them with a child with physical chronic disease and the other with a child without this condition. The children were of both sexes, 3 to 4 years old. The participants were selected in public hospitals and schools. A semi-structured interview was used to investigate the mother's childrearing practices, examined by content analysis. The results show a marginally significant difference showing that the group of mothers of the chronic disease children uses coercive practices less frequently. Moreover, there was a significant difference between the groups in relation to the use of physical punishment and privation/penalty, suggesting that mothers of chronic disease children use less frequently this type of practice. The results support the initial hypothesis that mothers of chronic disease children would be less coercive when compared to mothers of the other group.
\end{abstract}

Key words: childrearing practices; physical chronic disease; childhood

A interação mãe-criança e suas implicações para o processo de socialização infantil constituem um tópico de grande interesse das pesquisas em psicologia do desenvolvimento. Um dos principais aspectos investigados acerca da interação da díade refere-se às estratégias empre- gadas pela mãe na regulação do comportamento da criança. Alguns estudos apontam que a presença de doença crônica orgânica na infância pode representar um importante fator de mediação da qualidade da interação mãe-criança (Leicth, 1999; Posada, Carbonell, Alzate, Bustamante \& Arenas, 1999). Nes- 
te contexto, a condição de saúde da criança também tem implicações importantes na determinação das práticas educativas utilizadas pelas mães no controle e orientação do comportamento dos filhos (Ievers, 1998; Noll, McKellop, Vannatta \& Kalinyak, 1998).

A doença crônica tem sido definida como perturbações de saúde que persistem por longos períodos de tempo, podendo se estender ao longo de toda vida (Eiser, 1990). Perrin e Shonkoff (2000) acrescentam que tais doenças podem ser manejadas ou controladas no intuito de diminuir o sofrimento e melhorar a qualidade de vida dos doentes, porém na maioria dos casos não são completamente curadas. As hospitalizações extensivas, mesmo no período de diagnóstico, também são freqüentes. Apesar de muitas doenças crônicas serem consideradas relativamente raras, juntas, elas afetam cerca de 15 a $18 \%$ da população infantil. Deste total, aproximadamente 5\% seriam de doenças crônicas orgânicas persistentes ou recorrentes (Garralda, 1994).

O presente estudo enfocou especificamente a doença crônica orgânica (e.g., fibrose cística, disfunção renal crônica, cardiopatias e câncer). Dessa forma, foram excluídas outras formas de doença crônica como deficiências físicas (e.g., deformidades e falta de algum membro do corpo), dificuldades de aprendizagem e enfermidades neurológicas (e.g., déficit de atenção e epilepsia), doenças psicossomáticas (e.g., asma e obesidade) e doenças mentais (e.g., autismo e retardo mental).

A maneira como a criança responde à presença de uma doença crônica dependerá, segundo alguns autores (Eiser, 1990; Heinzer, 1998) de fatores relacionados à própria doença (e.g., limitações físicas e sociais que acarreta, diagnóstico precoce ou tardio, prognóstico, gravidade, visibilidade e origem genética); relacionados à criança (e.g., idade, gênero, temperamento e personalidade); e relacionados à família (e.g., estrutura familiar, habilidades de comunicação e solução de problemas). Assim, as implicações de longo prazo da doença crônica orgânica sobre o desenvolvimento cognitivo, social e emocional também diferem consideravelmente dependendo da interação desses vários fatores (Query, Reichelt \& Christoferson, 1990; Wasserman, 1992). A adequação das respostas dos cuidadores com relação à criança enferma está circunscrita, dentre outros aspectos, à sua percepção acerca da gravidade das condições de saúde da criança (Hamlett, Pellegrini \& Katz, 1992). Por exemplo, em crianças acometidas de câncer, Françoso e Valle (1999) referem que o primeiro ano de tratamento é o período de maior estresse para a criança e seus pais, devido principalmente ao diagnóstico recente e à ansiedade do início do tratamento.

A revisão da literatura realizada por Castro e Piccinini (2002) mostra certa consistência nos achados sobre doença crônica orgânica na infância, indicando que essas crianças possuem maiores riscos de apresentarem desajuste emocional, comportamental e social. Por exemplo, crianças com artrite reumatóide têm sido descritas como altamente sensíveis a críticas, à frustração, e demonstram auto-imagem empobrecida (Gortmaker, Walker, Wertzman \& Sobol, 1990). Em crianças com diabetes verificou-se uma relação entre o nível de con- trole glicêmico e ajustamento social (Fonagy, Moran, Linsay, Kurtz \& Borwn, 1987). Estudos com crianças portadoras de disfunção renal também indicaram uma relação entre desajustamento social e a gravidade da doença (Garralda, Jameson, Reynolds \& Postlehwaite, 1988). Hopkins, Roizen, Lindsay e Carrol (1995) encontraram em crianças com atresia de vias biliares, um tipo de doença hepática congênita, maiores riscos com relação ao temperamento, já que as deficiências nutricionais que acompanham a doença podem causar irritabilidade. Em crianças com fibrose cística foram encontrados altos índices de problemas de comportamento em pesquisa que incluía mais de uma enfermidade no grupo clínico (Canning, 1996). De modo análogo, o estudo de Heller, Rafman, Zvagulis e Pless (1985) evidenciou que as crianças portadoras de enfermidades congênitas, como, por exemplo, doenças cardíacas, apresentavam significativamente mais problemas de comportamento do que o grupo controle de crianças sem problemas de saúde. A investigação de Goldberg, Gotowiec e Simons (1995) apresentou resultados similares, indicando que crianças com doença cardíaca congênita e fibrose cística obtiveram índices mais altos de problemas de comportamento e índices mais altos de apego inseguro com seus cuidadores do que seus pares sem problemas de saúde.

No que concerne à relação da mãe e do pai com sua criança portadora de doença crônica, é comum que esses pais apresentem dificuldades em lidar com o seu filho. Tais complicações podem ser expressas através de comportamentos de extrema preocupação (Bradford, 1997), de irresponsividade às reais necessidades da criança (Leitch, 1999), de superproteção (Bradford, 1997; Sabbeth, 1984), de estilos interativos, ansioso e defensivo ou, ainda, de negligência, quando os pais procuram evitar o envolvimento com a criança por medo de que ela possa vir a morrer precocemente (Sabbeth, 1984). Nesse sentido, a presença de doenças ou deficiência física pode aumentar o risco de maus-tratos à criança (abuso ou negligência) no interior da família devido, em parte, ao estresse provocado pelo alto nível de exigência das crianças com precárias condições de saúde, e também, às experiências de separação precoce decorrentes de hospitalizações extensivas (White, Benedict, Wulff \& Kelley, 1987). Por outro lado, Espíndula e Valle (2002), referem que a dor e a experiência de ter um filho com câncer em estado grave não impede que mães e pais permaneçam firmes e fortes nos cuidados com a criança. No estudo realizado por Peçanha, Piccinini e Millar (2001), o tipo de doença crônica se mostrou um importante determinante do comportamento materno. A interação mãe-criança em díades com crianças asmáticas foi menos harmônica do que em díades cujas crianças sofriam de doença cardíaca congênita e crianças sem problemas de saúde. As mães de crianças com asma foram mais intrusivas e menos responsivas do que as mães dos outros dois grupos, com possíveis implicações na maneira pela qual a mãe lida com a autonomia e dependência com relação à criança com asma. A ausência de diferenças entre os dois últimos grupos sugere que não necessariamente a doença crônica se constitui num determinante da intrusividade e responsividade materna. Além disto, os resultados sugerem 
que existem particularidades entre as doenças crônicas que podem contribuir para comportamentos específicos da mãe.

Dessa forma, os pais de crianças cronicamente enfermas estão expostos a interações e situações muito estressantes, exigindo deles que lancem mão de práticas educativas para regular o comportamento da criança, muitas vezes alterado, em função das demandas médicas e de cuidados com a saúde. No estudo realizado por Ievers (1998) sobre práticas educativas empregadas por mães de crianças com fibrose cística entre 06 e 11 anos de idade, os resultados indicaram que as mães dessas crianças utilizavam mais punição e autoritarismo, ao mesmo tempo em que se sentiam menos satisfeitas no seu papel materno do que as mães de crianças sem problemas de saúde. De maneira similar, as crianças referiram uma atitude menos positiva em direção aos seus pais, reportaram o uso de autoritarismo materno e demonstraram pouca satisfação com a ajuda que suas mães forneciam com o regime de tratamento da fibrose cística. Entretanto, em outra investigação realizada por Noll, McKellop, Vannata e Kalinyak (1998), sobre práticas educativas em crianças enfermas a partir da perspectiva de seus cuidadores e de profissionais, os resultados não foram semelhantes. Os pesquisadores verificaram que os profissionais que trabalhavam com essas crianças possuíam idéias estereotipadas que não correspondiam às reais práticas educativas empregadas pelos cuidadores. Através de técnicas psicométricas verificou-se que os profissionais de saúde acreditavam que a conduta dos pais se caracterizaria por preocupações e disciplina excessivas, bem como por superproteção em relação à criança, o que não correspondeu aos relatos dos pais.

Embora os achados dos estudos revisados sejam por vezes controversos, a presença de doença crônica na criança se constitui em um importante organizador de respostas parentais com conseqüências para as suas interações com a criança. A enfermidade crônica na infância pode afetar profundamente a percepção dos pais acerca do desenvolvimento de seus filhos, bem como a forma com que a disciplina é introduzida e conduzida na vida da criança.

Com relação às práticas educativas utilizadas pelos pais no processo de socialização da criança, Hoffman $(1975,1994)$ apresenta um modelo teórico que prevê os efeitos de diferentes tipos de práticas sobre o desenvolvimento da criança. As práticas educativas parentais são definidas na literatura como estratégias utilizadas pelos pais para orientar o comportamento dos filhos no sentido de fazer com que eles adquiram certos comportamentos e, também, para suprimir ou reduzir outros comportamentos considerados socialmente inadequados ou desfavoráveis (Grusec \& Lytton, 1988; Mussen, Conger, Kagan \& Huston, 1990; Newcombe, 1999).

Para Hoffman $(1975,1994)$ as práticas educativas parentais podem ser agrupadas em duas grandes categorias distintas: as estratégias indutivas e as estratégias de força coercitiva. Ambos os tipos de estratégias têm por função comunicar à criança o desejo dos pais de que ela modifique seu comportamento, bem como pressionar a criança a obedecer. As estratégias indutivas caracterizam-se por atingir o objetivo disciplinar indicando para a criança as conseqüências do seu com- portamento para as outras pessoas e chamando sua atenção para os aspectos lógicos da situação. Isso propicia à criança a compreensão das implicações de suas ações e, portanto, dos motivos que justificam a necessidade de mudança no seu comportamento. Desta forma, a criança desenvolve certa autonomia para utilizar esse tipo de informação para controlar seu próprio comportamento. Como exemplos desse tipo de estratégia pode-se citar o uso de explicações sobre o comportamento da criança e as suas conseqüências e explicações a respeito de regras. Esse tipo de estratégia tende a facilitar a internalização de padrões morais (Hart, Ladd \& Burleson, 1990; Hoffman, 1991, 1994).

Por outro lado, as estratégias de força coercitiva caracterizam-se, segundo Hoffman (1975, 1994), pela aplicação direta da força, incluindo punição física, privação de privilégios e afeto ou pelo uso de ameaças. Essas técnicas fazem com que a criança controle seu comportamento em função das reações punitivas dos pais. Além disso, elas produzem emoções intensas tais como medo, raiva e ansiedade, que tendem a reduzir ainda mais a possibilidade de a criança compreender a situação e a necessidade de modificação de comportamento. Ou seja, o controle do comportamento da criança tenderá a depender de intervenções externas porque ela não adquire a capacidade de compreender as implicações de suas ações. Nesse sentido, as estratégias de força coercitiva não favorecem a internalização das regras sociais e padrões morais.

Muitos estudos têm investigado as implicações desses diferentes tipos de práticas educativas para o desenvolvimento infantil. A maioria deles tem demonstrado de forma bastante consistente que, enquanto as estratégias de caráter indutivo encontram-se associadas ao desenvolvimento de padrões de comportamento ajustados, competência social e cooperação (Baumrind, 1966; Baumrind \& Black, 1967; Robinson, Mandleco, Olsen \& Hart, 1995), as estratégias de força coercitivas tendem a estar relacionadas a problemas de comportamento (Mounts, Steinberg \& Dornbusch, 1991; Rothbaum \& Weisz, 1994).

Devido à importância das práticas educativas parentais sobre o desenvolvimento infantil, vários pesquisadores têm se preocupado em identificar os determinantes dessas práticas. A literatura apresenta um certo consenso em relação à noção de que as estratégias utilizadas pelos pais no processo de socialização da criança são uma função de inúmeros fatores interatuantes, dentre estes a condição de saúde da criança tem sido referida como um importante fator de mediação da qualidade da interação mãe-criança (Bradford, 1997; Sabbeth, 1984; White \& cols., 1987). Neste campo de investigação, constata-se, em especial, um crescente interesse na investigação do impacto da doença crônica orgânica infantil sobre o sistema familiar bem como sobre a própria criança (Berenbaum \& Hatcher, 1992; Burke \& Elliot, 1999; Canning, Harris \& Kelleher, 1996; Heinzer, 1998; Silver, Westbrook \& Stein, 1998; Wallander \& Varni, 1998). Entretanto, os estudos sobre o impacto da doença crônica infantil sobre a relação mãe-criança tem gerado achados inconsistentes. Soma-se a isto o fato de existirem poucos estudos enfocando, especificamente, as práticas educativas maternas em situações que 
envolvam perturbações da saúde infantil. Essa situação aponta para a necessidade de se investigar as práticas educativas utilizadas pelas mães de crianças cronicamente doentes, enquanto um importante indicador do processo de socialização. Neste sentido, o presente estudo teve por objetivo investigar as implicações da doença crônica infantil nas práticas educativas maternas. Para tanto, buscou-se comparar as práticas educativas relatadas por mães de crianças com doença crônica orgânica com as práticas utilizadas por mães de crianças sem doença crônica. Tendo em vista que alguns estudos já mostraram que as mães de crianças com doença crônica tendem a ser mais protetoras do que mães de crianças sem doença crônica (Bradford, 1997; Sabbeth, 1984), esperava-se que as mães do primeiro grupo, quando comparadas com as demais, utilizassem mais praticas educativas indutivas do que coercitivas.

\section{Método}

\section{Participantes}

Participaram do estudo 40 mães, metade das quais eram mães de crianças portadoras de doença crônica orgânica (Grupo 1) e as demais de crianças sem doença crônica (Grupo 2). Todas as mães eram casadas ou viviam com o companheiro. As crianças eram de ambos os sexos e tinham entre 3 e 5 anos de idade. As mães do grupo de doentes crônicos tinham idade média de 30,1 anos (DP = 5,3), com escolaridade média de 8,9 anos ( $D P=2,3$ ). Neste grupo, 13 meninos e 7 meninas apresentavam algum tipo de doença crônica orgânica, dentre elas: leucemia, tumores, insuficiência renal, insuficiência hepática e fibrose cística. As mães do grupo sem doença crônica tinham idade média de 33,0 anos (DP = 7,5), com escolaridade média de 9,7 anos (DP = 1,9). Neste grupo havia 11 meninos e 9 meninas que não apresentavam problemas de saúde crônicos de acordo com avaliação feita com a mãe da criança antes da entrevista. Como havia mais meninos do que meninas com doença crônica, buscou-se emparelhar este grupo ao Grupo 1, pelo sexo da criança. Assim ambos os grupos foram compostos por mais meninos (Grupo $1=65 \%$, Grupo 2 =55\%) do que meninas (Grupo $1=35 \%$, Grupo 2 = 45\%). A Tabela 1 apresenta detalhes dos participantes e resultados do teste Mann-Whitney utilizado para analisar eventuais diferenças entre os grupos.

Como pode ser visto na Tabela, com relação às crianças, não houve diferenças significativas entre os grupos em nenhum dos indicadores de desenvolvimento investigados (primeiros passos, primeiras palavras e controle dos esfíncteres). Em relação ao número de hospitalizações, como era esperado, houve uma diferença significativa $(p<0,01)$ entre os grupos, revelando que as crianças com doença crônica tiveram

Tabela 1

Dados demográficos dos participantes e do desenvolvimento das crianças em cada grupo

\begin{tabular}{|c|c|c|c|c|}
\hline & $\begin{array}{l}\text { Com doença crônica } \\
\qquad(\mathrm{n}=20)\end{array}$ & $\begin{array}{l}\text { Sem doença crônica } \\
(n-20)\end{array}$ & $U$ & $p$ \\
\hline \multicolumn{5}{|l|}{ Sexo das crianças } \\
\hline Masculino & $13(65 \%)$ & $11(55 \%)$ & & \\
\hline Feminino & $07(35 \%)$ & $09(45 \%)$ & & \\
\hline \multicolumn{5}{|c|}{ Idade das crianças $^{1}$} \\
\hline $\mathrm{M}$ & 47,70 & 49,20 & \multirow{2}{*}{0,30} & \multirow{2}{*}{0,59} \\
\hline DP & 7,99 & 9,75 & & \\
\hline \multicolumn{5}{|c|}{ Tempo de amamentação } \\
\hline M & 6,20 & 11,30 & \multirow[b]{2}{*}{1,04} & \multirow[b]{2}{*}{0,31} \\
\hline DP & 6,41 & 21,42 & & \\
\hline \multicolumn{5}{|c|}{ Primeiros passos da criança } \\
\hline $\mathrm{M}$ & 14,26 & 12,28 & \multirow{2}{*}{1,60} & \multirow{2}{*}{0,21} \\
\hline DP & 5,84 & 3,27 & & \\
\hline \multicolumn{5}{|c|}{ Primeiras palavras da } \\
\hline criança & 11,28 & 12,63 & \multirow{2}{*}{0,35} & \multirow{2}{*}{0,56} \\
\hline $\begin{array}{l}\mathrm{M} \\
\mathrm{DP}\end{array}$ & 6,48 & 6,88 & & \\
\hline \multicolumn{5}{|c|}{ Controle dos esfíncteres } \\
\hline $\mathrm{M}$ & 19,76 & 19,61 & \multirow{2}{*}{0,003} & \multirow{2}{*}{0,95} \\
\hline DP & 9,16 & 6,45 & & \\
\hline \multicolumn{5}{|l|}{ Idade das mães $^{2}$} \\
\hline $\mathrm{M}$ & 30,15 & 33,00 & \multirow{2}{*}{1,85} & \multirow{2}{*}{0,18} \\
\hline DP & 5,35 & 7,47 & & \\
\hline \multicolumn{5}{|c|}{ Escolaridade das mães } \\
\hline $\mathrm{M}$ & 8,95 & 9,68 & \multirow{2}{*}{1,24} & \multirow{2}{*}{0,27} \\
\hline DP & 2,33 & 1,88 & & \\
\hline
\end{tabular}


maior número de hospitalizações do que as do outro grupo. Quanto aos fatores relacionados à mãe, nenhuma diferença significativa foi encontrada entre os dois grupos.

\section{Delineamento e Procedimentos}

Foi utilizado um delineamento de grupos contrastantes (Nachmias \& Nachmias, 1996), buscando comparar as práticas educativas utilizadas pelas mães de crianças com e sem doença crônica orgânica. As mães do grupo de crianças doentes crônicas foram contatadas por ocasião das re-consultas agendadas nos hospitais que participaram do presente estudo. No mesmo local foi inicialmente realizada a entrevista de avaliação do desenvolvimento e da saúde da criança (GIDEP, 1994a). Esta entrevista adaptada de Peçanha e colaboradores (2001), teve por objetivo avaliar os indicadores de desenvolvimento e as condições de saúde da criança. As mães foram solicitadas a dar informações sobre a alimentação da criança, desenvolvimento psicomotor, linguagem, controle dos esfíncteres, sintomas físicos e estado de saúde da criança. Os dados permitiram confirmar as informações dos prontuários da amostra de crianças doentes crônicas, bem como caracterizar a amostra de crianças sem doença crônica. Logo após era realizada a entrevista sobre estratégias maternas de regulação do comportamento infantil (GIDEP, 1994b). Esta entrevista foi desenvolvida para examinar as práticas educativas utilizadas pelas mães, tendo por base a entrevista desenvolvida por Piccinini, Maggi e Carro (1993) e Hart e colaboradores (1990). A entrevista era composta de duas partes. Inicialmente as mães eram solicitadas a mencionar situações cotidianas em que elas achavam difícil de lidar com a criança, bem como relatar o que faziam em tais situações. $\mathrm{Na}$ segunda parte, eram apresentadas seis situações hipotéticas envolvendo situações de conflito moral, que implicam o uso de práticas educativas maternas. As situações faziam referência a uma criança, da mesma idade e sexo do(a) filho(a) cuja mãe estava sendo entrevistada. As situações eram: a) criança recusa-se a vestir a roupa escolhida pela mãe; b) criança recusa-se a almoçar; c) criança leva para casa um brinquedo de seu amigo sem seu consentimento; d) criança pinta com canetas as paredes da sala da sua casa; e) criança recusa-se a tomar banho; f) criança agride outra criança. Após a apresentação de cada situação hipotética, solicitava-se que a mãe relatasse se isto já havia acontecido com sua criança. Caso a resposta fosse positiva, solicitava-se que a mãe contasse o que havia feito em tal situação. Se a resposta fosse negativa, perguntava-se o que ela faria se isto ocorresse. Para fins do presente estudo somente a segunda parte da entrevista, envolvendo as situações hipotéticas, foi analisada. As mesmas entrevistas também foram utilizadas com as mães do grupo de crianças sem doença crônica, que foram contatadas e entrevistadas na própria pré-escola da sua criança.

\section{Resultados}

Análise de conteúdo quantitativa (Bardin, 1977) foi utilizada para examinar as práticas educativas relatadas pelas mães nas entrevistas em relação às seis situações investigadas. Com base nos trabalhos de Hoffman (1975; 1994) e Alvarenga e Piccinini (2001), desenvolveu-se uma estrutura de dez categorias que foi utilizada para classificar as práticas educativas maternas em indutivas, coercitivas e não-interferência. Nove categorias foram agrupadas em dois grandes grupos: a) práticas indutivas 1) negociação/troca; 2) explicação apontando conseqüências; 3) explicação baseada em convenções; 4) comando verbal não-coercitivo; e b) práticas coercitivas: 5) punição verbal; 6) ameaça de punição; 7) privação/castigo; 8) coação física; 9) punição física. A décima categoria que foi denominada não-interferência envolvia práticas que não foram classificadas nas categorias acima. A classificação das práticas educativas relatadas pelas mães foi realizada por dois codificadores que tiveram treinamento extensivo (12 horas) e que desconheciam o grupo a que cada mãe pertencia. O índice de concordância entre os codificadores foi calculado sobre uma amostra de 30\% das entrevistas e atingiu o valor de 85\%. Uma vez tendo obtido este índice de concordância, todas as respostas foram classificadas independentemente pelos dois juizes. Um terceiro juiz foi utilizado para dirimir as discordâncias.

Tendo em vista o objetivo do presente estudo de examinar eventuais diferenças nas práticas educativas entre os dois grupos, as análises que seguem foram feitas em relação ao conjunto das seis situações. Isto também ocorreu em função da baixa freqüência de algumas categorias de práticas, caso fossem analisadas para cada situação. Dos 439 relatos de práticas educativas maternas, 58\% (254) foram relatadas pelas mães do grupo sem doença crônica e 42\% (185) pelo grupo com doença crônica. A Tabela 2 apresenta os percentuais de resposta dos dois grupos em cada categoria de prática educativa nas seis situações investigadas.

Como pode ser visto na Tabela 2, as análises não revelaram diferenças expressivas no total de práticas indutivas e coercitivas mencionadas pelas mães dos dois grupos. As práticas indutivas foram um pouco mais mencionadas pelas mães do grupo com doença crônica (64\%) do que pelas mães do grupo sem doença crônica (59\%). O contrário foi constatado quanto às práticas coercitivas, que foram mais referidas pelo grupo sem doença crônica (35\%) do que pelo outro grupo (30\%). Quanto às categorias específicas que compõem as práticas indutivas e coercitivas, também não foram verificadas diferenças marcantes entre os grupos.

Para examinar eventuais diferenças na tendência para utilizar práticas indutivas ou coercitivas foi utilizado o teste de Mann-Whitney nos dados sobre a incidência média das freqüências de cada prática educativa. A Tabela 3 apresenta a incidência média, desvio padrão, valor de $U$ e o nível de significância das práticas educativas mencionadas pelas mães.

Examinando-se as médias do total de práticas educativas mencionadas, verifica-se que as mães do grupo com doença crônica $(\mathrm{M}=9,25)$ relataram um número significativamente menor de práticas $(p<0,05)$ em relação ao grupo sem doença crônica $(\mathrm{M}=12,70)$. Os resultados também revelaram uma diferença marginalmente significativa quanto à média de prá- 
Tabela 2

Percentuais de resposta dos dois grupos em cada categoria de prática educativa nas seis situações investigadas

\begin{tabular}{lcc}
\hline \multicolumn{1}{c}{ Práticas educativas maternas } & Com doença crônica & Sem doença crônica \\
\hline 1. Não interferência & $5 \%(10)$ & $6 \%(15)$ \\
2. Negociação/conversa & $23 \%(42)$ & $16 \%(41)$ \\
3. Explicação/conseqüência & $10 \%(19)$ & $13 \%(32)$ \\
4. Explicação/convenção & $15 \%(28)$ & $14 \%(37)$ \\
5. Comando verbal não coercitivo & $16 \%(30)$ & $15 \%(39)$ \\
6. Punição verbal & $16 \%(30)$ & $13 \%(34)$ \\
7. Ameaça de punição & $2 \%(03)$ & $3 \%(08)$ \\
8. Privação/castigo & $4 \%(08)$ & $7 \%(09)$ \\
9. Coação física & $5 \%(10)$ & $6 \%(15)$ \\
10. Punição física & $3 \%(05)$ & $5 \%(14)$ \\
\hline Total de práticas indutivas & $64 \%(119)$ & $59 \%(149)$ \\
\hline Total de práticas coercitivas & $30 \%(56)$ & $35 \%(90)$ \\
\hline Total geral de práticas & $(185)$ & $(254)$ \\
\hline
\end{tabular}

Nota: total de práticas nos dois grupos: 439

Tabela 3

Incidência média, desvio padrão, valor de U e nível de significância (teste de Mann-Whitney) das práticas educativas maternas para cada grupo nas seis situações investigadas

\begin{tabular}{|c|c|c|c|c|}
\hline Práticas educativas maternas & $\begin{array}{c}\text { Com doença crônica } \\
\text { Média (DP) }\end{array}$ & $\begin{array}{c}\text { Sem doença crônica } \\
\text { Média (DP) }\end{array}$ & $U$ & $p$ \\
\hline 1) Não-interferência & $0,50(0,69)$ & $0,75(0,72)$ & 1,19 & 0,23 \\
\hline 2) Negociação/conversa & $2,10(1,33)$ & $2,05(1,05)$ & 0,21 & 0,83 \\
\hline Explicação/conseqüência & $0,95(1,19)$ & $1,60(1,35)$ & 1,59 & 0,11 \\
\hline Explicação/convenção & $1,40(1,05)$ & $1,85(1,66)$ & 0,80 & 0,42 \\
\hline Comando verbal não- & $1,50(0,83)$ & $1,95(1,05)$ & 1,31 & 0,18 \\
\hline coercitivo & $1,50(1,24)$ & $1,70(1,49)$ & 0,30 & 0,75 \\
\hline 6) Punição verbal & $0,15(0,37)$ & $0,40(0,75)$ & 1,17 & 0,24 \\
\hline 7) Ameaça de punição & $0,40(0,82)$ & $0,95(1,05)$ & 2,10 & 0,03 \\
\hline 8) Privação/castigo & $0,50(0,76)$ & $0,75(0,85)$ & 1,07 & 0,34 \\
\hline $\begin{array}{l}\text { 9) Coação física } \\
\text { 10) Punição física }\end{array}$ & $0,25(0,44)$ & $0,70(0,80)$ & 1,89 & 0,05 \\
\hline Total de práticas indutivas & $5,95(2,11)$ & $7,45(2,99)$ & 1,46 & 0,14 \\
\hline Total de práticas coercitivas & $2,80(1,43)$ & $4,50(3,54)$ & 1,72 & 0,08 \\
\hline Total geral de práticas & $9,25(2,69)$ & $12,70(4,69)$ & 2,80 & 0,05 \\
\hline
\end{tabular}

ticas coercitivas mencionadas pelos dois grupos $(p<0,08)$. O grupo com doença crônica apresentou uma incidência média menor de práticas coercitivas $(\mathrm{M}=2,80)$ do que o grupo sem doença crônica $(M=4,50)$. Tendência oposta, mas não significativa $(p<0,14)$ ocorreu com o total de práticas indutivas (grupo com doença crônica: $M=5,95$; grupo sem doença crônica: $\mathrm{M}=7,45)$.
Algumas diferenças significativas ocorreram com relação às categorias de práticas educativas que compõem as práticas coercitivas. Houve uma diferença significativa na categoria privação/castigo $(p<0,03)$, indicando que as mães do grupo com doença crônica $(M=0,40)$ apresentaram uma incidência média menor de respostas nesta categoria do que as mães de crianças do grupo sem doença crônica $(\mathrm{M}=0,95)$. 
Outra diferença significativa foi constatada na categoria punição física $(p<0,05)$, revelando que as mães do grupo com doença crônica $(\mathrm{M}=0,25)$ apresentaram uma incidência media menor nesta categoria, quando_comparadas às mães do grupo sem doença crônica $(\mathrm{M}=0,70)$. Nas demais categorias de práticas coercitivas e indutivas não houve diferenças significativas.

\section{Discussão}

O objetivo do presente estudo foi o de investigar eventuais particularidades nas práticas educativas de mães de crianças portadoras de doença crônica orgânica. Os resultados apóiam parcialmente a expectativa inicial de que as mães do grupo com doença crônica utilizariam menos práticas coercitivas do que as mães do grupo sem doença crônica. Por outro lado, não foram encontradas evidências que apoiassem a expectativa de que as mães do grupo com doença crônica utilizariam mais práticas indutivas do que as mães do outro grupo.

Os resultados revelaram uma diferença marginalmente significativa relacionada ao conjunto das práticas coercitivas relatadas, mostrando que as mães de crianças portadoras de doença crônica mencionaram, de modo geral, menos esse tipo de prática nas situações hipotéticas investigadas. Esse achado corrobora a literatura a respeito do tema (Noll et al., 1998), que enfatizou que a condição física da criança pode influenciar as práticas educativas empregadas, fazendo com que a mãe evite o uso da coerção e lance mão de outras estratégias. Examinando especificamente os diferentes tipos de práticas coercitivas, nas categorias punição física e privação/castigo foram reveladas diferenças significativas entre os grupos. As mães do grupo de doentes crônicos mencionaram significativamente menos o uso destas duas categorias, quando comparadas com o outro grupo. Dentre as diferentes práticas coercitivas relatadas pelas mães no presente estudo, a punição física e a privação/castigo podem ser consideradas mais severas se comparadas às demais (punição verbal, ameaça de punição e coação física). A punição física envolve o uso da força para causar dor à criança e o uso de castigos ou privações tendem a causar humilhações ou a perda de algo importante para a criança (Hoffman, 1975, 1994). O uso pouco freqüente dessas práticas pelas mães de crianças com doença crônica, pode estar relacionado à fragilidade física de seus filhos, que são submetidos freqüentemente a procedimentos dolorosos e a um tratamento médico rigoroso. A pouca utilização de práticas coercitivas seria uma forma de poupar a criança de mais sofrimentos. É possível também que, por características da própria doença da criança, as mães tenham receio de que práticas que envolvam o uso da força possam trazer conseqüências nocivas à saúde de seu filho. Uma criança leucêmica, por exemplo, é mais vulnerável a ferimentos causados pelo uso da coação ou da punição física (palmada, beliscão), o que pode trazer conseqüências graves para a sua saúde. Quanto aos aspectos subjetivos das mães, a dor de ver o filho doente (Espíndula \& Valle, 2002) somada à extrema preocupação e até à superproteção do filho (Bradford, 1997;
Sabbeth, 1984) podem, da mesma forma, estar interferindo na sua conduta de poupar a criança da dor física e psicológica que representa o uso da punição física e da privação/castigo.

Quanto à expectativa inicial de que haveria maior uso de práticas indutivas por parte das mães do grupo de crianças com doença crônica, os resultados não revelaram diferenças significativas entre os grupos. Os dados sugerem que o uso menos freqüente de práticas coercitivas não implica, necessariamente, o aumento da utilização de práticas indutivas. Assim, no presente estudo, tanto as mães de crianças com doença crônica quanto as mães do outro grupo relataram que utilizavam negociações, explicações e comandos verbais nãocoercitivos com uma freqüência semelhante. Contudo, é interessante assinalar que a média geral de práticas empregadas pelas mães de crianças com doença crônica foi significativamente inferior à média geral do outro grupo. Esse dado pode estar indicando que as mães de crianças com doença crônica utilizam um repertório menor de práticas educativas em função das restrições impostas pela condição de saúde dos filhos. Outra explicação possível diz respeito ao comportamento da própria criança. Nesse caso, crianças com doença crônica seriam mais obedientes ou cooperativas em relação às solicitações maternas, demandando das mães menos estratégias para regular o seu comportamento. No entanto, essas questões devem ser melhor investigadas em futuras pesquisas.

De acordo com a literatura, as práticas coercitivas são menos favoráveis para o desenvolvimento infantil, estando associadas, freqüentemente, a problemas de comportamento e déficits no repertório de competência social. Nesse sentido, a baixa incidência de práticas coercitivas no grupo crônico, revelada no presente estudo, tenderia a trazer benefícios para o desenvolvimento da criança com doença crônica. Todavia, vários estudos apontam que essas crianças teriam maior probabilidade de desenvolver problemas de comportamento do que crianças sem problemas de saúde (Canning, 1996; Heller et al., 1985). Embora a incidência de problemas de comportamento não tenha sido aqui examinada, é possível que os problemas de comportamento entre crianças com doença crônica estejam mais associados à permissividade dos pais também decorrente dos problemas de saúde da criança, do que à utilização de práticas coercitivas. Para esclarecer esta questão são necessários novos estudos que examinem os dois construtos, práticas educativas e problemas de comportamento em grupos portadores de doença crônica.

O presente estudo explorou alguns aspectos dessa problemática através dos relatos das próprias mães sobre suas praticas educativas. Esta abordagem poderá ser enriquecida por estudos observacionais que examinem situações de interação mãe-criança, especialmente no contexto familiar, o que permitirá incluir não só o pai, mas outros membros da família que convivam com a criança com doença crônica. Juntos, esses estudos contribuirão para se compreender as particularidades das práticas educativas utilizadas pelos pais de crianças enfermas crônicas. Isto tem implicações tanto teóricas como práticas, ao contribuir para que os pais de crianças 
com doença crônica sejam auxiliados a lidar com o dia-a-dia de uma criança que, além de apresentar necessidades próprias do seu desenvolvimento, que implicam muitas vezes na busca de autonomia e resistência à autoridade parental, precisa também atender as demandas médicas por medicação e cuidados especiais.

\section{Referências}

Alvarenga, P., \& Piccinini, C. (2001). Práticas educativas maternas e problemas de comportamento em pré-escolares. Psicologia: Reflexão e Crítica, 14(3), 449-460.

Bardin, L. (1977). Análise de conteúdo (L. A. Reto \& A. Pinheiro, Trad.). São Paulo: Martins Fontes.

Baumrind, D. (1966). Effects of authoritative parental control on child behavior. Child Development, 37, 887-907.

Baumrind, D., \& Black, A. E. (1967). Socialization practices associated with dimensions of competence in preschool boys and girls. Child Development, 38, 291-327.

Berenbaum, J., \& Hatcher, J. (1992). Emotional distress of mothers of hospitalized children. Journal of Pediatric Psychology, 17(3), 359-372.

Bradford, R. (1997). Children, families and chronic disease. Londres: Routdlege.

Burke, P., \& Elliott, M. (1999). Depression in pediatric chronic illness: a diathesis-stress model. Psychosomatics, 40(1), 5-17.

Canning, R. D., Harris, E. S., \& Kelleher, K. J. (1996). Factor predicting distress among caregivers to children with chronic medical conditions. Journal of Pediatric Psychology, 21(5), 735-749.

Castro, E. K., \& Piccinini, C. A. (2002). Implicações da doença orgânica crônica na infância para as relações familiares: algumas questões teóricas. Psicologia: Reflexão e Critica, 15(3), 625-635.

Eiser, C. (1990). Chronic childhood disease. Nova York: Cambridge.

Espíndula, J. A., \& Valle, E. R. M. (2002). Experiência materna diante da iminência de morte do filho com recidiva de câncer. Pediatria Moderna, 38(5), 188-194.

Fonagy, P., Moran, G. S., Linsay, M. K. M., Kurtz, A. B., \& Borwn, R.(1987). Psychological adjustment and diabetics control. Archives of Disease in Childhood, 62, 1009-1013.

Françoso, L. P. C., \& Valle, E. R. M. (1999). A criança com câncer - estudo preliminar. Pediatria Moderna, 35(5), 320-331.

Garralda, M. E. (1994). Chronic physical illness and emotional disorder in childhood. British Journal of Psychiatry, 164, 8-10.

Garralda, M. E., Jameson, R. A., Reynolds, J. M., \& Postlethwaite, J. R. (1988). Psychiatric adjustment in children with chronic renal failure. Child Psychology and Psychiatric, 29, 79-90.

Grupo de Interação Social, Desenvolvimento e Psicopatologia - GIDEP (1994a). Entrevista de Avaliação do Desenvolvimento e da Saúde da Criança. Instrumento não-publicado, Universidade Federal do Rio Grande do Sul, Porto Alegre.

Grupo de Interação Social, Desenvolvimento e Psicopatologia - GIDEP (1994b). Entrevista sobre estratégias maternas de regulação do comportamento infantil. Instrumento não-publicado, Universidade Federal do Rio Grande do Sul, Porto Alegre.

Goldberg, S., Gotowiec, A., \& Simons, R. (1995). Infant-mother attachment and behavior problems in healthy and chronically ill preschoolers. Development and Psychopathology, 7, 267-282.

Gortmaker, S. L., Walker, D. K., Weitzman, M., \& Sobol, A. M. (1990). Chronic conditions, socioeconomic risks, and behavioral problems in children and adolescents. Pediatrics, 85(3), 267-276.

Grusec, J. E., \& Lytton, H. (1988). Social development: history, theory and research. Nova York: Springer-Verlag.
Hamlett, K. W., Pellegrini, D. S., \& Katz, K. (1992). Childhood chronic illness as a family stressor. Journal of Pediatric Psychology, 17(1), 33-47.

Hart, C. H., Ladd, G. W., \& Burleson, B. R. (1990). Children’s expectations of the outcomes of social strategies: relations with sociometric status and maternal disciplinary styles. Child Development, 61, 127-137.

Heinzer, M. M. (1998). Health promotion during childhood chronic illness: a paradox facing society. Holistic Nursing Practice, 12(2), 8-16.

Heller, A. R., Rafman, S., Zvagulis, I., \& Pless, I. B. (1985). Birth defects and psychosocial adjustment. American Journal of Diseases of Children, 139, 257-263.

Hoffman, M. L. (1975). Moral internalization, parental power, and the nature of parent-child interaction. Developmental Psychology, 11, 228-239.

Hoffman, M. L. (1991). Commentary. Human Development, 34, 105-110.

Hoffman, M. L. (1994). Discipline and internalization. Developmental Psychology, 30, 26-28.

Hopkins, J., Roizen, N. J., Lindsay, D., \& Carrol, R. (1995). Development and treatment of infants with biliary atresia. Children's Health Care, 24(2), 97106.

Ievers, C. E. (1998). Maternal child-rearing behavior in cystic fibrosis. Dissertation Abstracts International, 58(10-B), 5646.

Leitch, D. B. (1999). Mother-infant interaction: achieving synchrony. Nursing Research, 49(1), 55-58.

Mounts, S. D., Steinberg, L., \& Dornbusch, S. (1991). Patterns of competence and adjustment among adolescents from authoritative, authoritarian, indulgent and neglectful families. Child Development, 62, 1049-1065.

Mussen, P. H., Conger, J. J., Kagan, J., \& Huston, A. C. (1990). Child development \& personality. Nova York: Harper \& Row.

Nachmias, C., \& Nachmias, D. (1996). Research methods in the social sciences. Londres: Arnold.

Newcombe, N. (1999). Desenvolvimento infantil: abordagem de Mussen (C. Buchweitz, Trad.). Porto Alegre: Artes Médicas (Original publicado em 1996).

Noll, R. B., McKellop, J. M., Vannatta, K., \& Kalinyak, K. (1998). Childrearing practices of primary caregivers of children with sickle cell disease: the perspective of professionals and caregivers. Journal of Pediatric Psychology, 23(2), 131-140.

Peçanha, D., Piccinini, C., \& Millar, S. (2001). Mother-child interaction with asthmatic, congenital hearth disease and healthy children. Manuscrito nãopublicado.

Perrin, J. M., \& Shonkoff, J. P. (2000). Developmental disabilities and chronic illness: an overview. In R. E. Behrman, R. M. Kliegnan \& H. B. Jenson (Orgs.), Nelson textbook of Pediatrics (16a ed., pp. 452-464). Filadélfia: W. B. Saunders.

Piccinini, C. A., Maggi, A., \& Carro, J. (1993). Strategies used by mothers of German and Italian descent for regulating their children behavior. Trabalho apresentado na XXII Biennal Meeting, International Society for the Study of Behavior Development, Recife, Brasil.

Posada, G., Carbonel, O. A., Alzate, G., Bustamante, M. R, \& Arenas, A. (1999). Maternal care and attachment security in ordinary and emergency contexts. Developmental Psychology, 35(6), 1370-1388.

Query, J. M., Reichelt, C., \& Christoferson, L. A. (1990). Living with chronic illness: a retrospective study of patients shunted for hydrocephalus and their families. Developmental Medicine and Child Neurology, 32, 119-128.

Robinson, C. C., Mandleco, B., Olsen, S. F., \& Hart, C. H. (1995). Authoritative, authoritarian and permissive parenting practices: development of a new measure. Psychological Reports, 77, 819-830.

Rothbaum, F., \& Weisz, J. R. (1994). Parental caregiving and child externalizing behavior in nonclinical samples: a meta-analysis. Psychological Bulletin, 116, 55-74.

Sabbeth, B. (1984). Understanding the impact of chronic childhood illness on families. Pediatric Clinics of North America, 31(1), 47-57. 
Silver, E. J., Westbrook, L. E., \& Stein, R. E. (1998). Relationship of parental psychological distress to consequences of chronic health conditions in children. Journal of Pediatric Psychology, 23(1), 5-15.

Wallander, J. L., \& Varni, J. W. (1998). Effects of pediatric chronic physical disorders on child and family adjustment. Journal of Child Psychology and Psychiatry, 39(1), 29-46.
Wasserman, M. D. A. (1992). Princípios de tratamento psiquiátrico de crianças e adolescentes com doenças físicas. In B. Garfinkel, G. Carlson, E. Weller (Orgs.), Transtornos psiquiátricos da infância e adolescência. Porto Alegre: Artes Médicas.

White, R., Benedict, M., Wulff, L., \& Kelley, M. (1987). Physical disabilities as risk factors for child maltreatment: a selected review. American Journal of Orthopsychiatric, 57(1), 93-101.

1 Agradecemos a colaboração de Elena Ione Panzenhagen (in memoriam) durante a fase de coleta dos dados, bem como à FAPERGS e ao CNPq pelo auxílio financeiro.

Cesar Augusto Piccinini é psicólogo, doutor em Psicologia pela University College London, Inglaterra, professor do Instituto de Psicologia da Universidade Federal do Rio Grande do Sul (UFRGS) e pesquisador do CNPq.

Elisa Kern de Castro é psicóloga, mestre em Psicologia do Desenvolvimento pela UFRGS e doutoranda em Psicologia Clínica e da Saúde pela Universidad Autónoma de Madrid, Espanha (bolsista CAPES).

Patrícia Alvarenga é psicóloga, mestre e doutoranda em Psicologia do Desenvolvimento pela UFRGS e professora da Universidade Luterana do Brasil (ULBRA).

Sílvia Vargas é psicóloga, especialista em Psicologia do Desenvolvimento pela UFRGS.

Viviane Ziebel Oliveira é psicóloga do Hospital de Clínicas de Porto Alegre, mestre e doutora em Psicologia do Desenvolvimento pela UFRGS.

Endereço para correspondência: Instituto de Psicologia, Universidade Federal do Rio Grande do Sul, Rua Ramiro Barcelos, 2600, Porto Alegre, RS, CEP 90035-003. E-mail: piccinini@portoweb.com.br 